\title{
Diagnostic Accuracy of Clinical Examination in Cervical Spine Injuries in Awake and Alert Blunt Trauma Patients
}

\author{
Manzar Hussain, Gohar Javed \\ Section of Neurosurgery, Department of Surgery, Aga Khan University Hospital, \\ Aga Khan University Medical College, Karachi, Pakistan
}

Study Design: Observational, case series.

Purpose: To determine the sensitivity and specificity of clinical judgment as compared to the use of X-ray images in detecting cervical spine injuries in trauma patients presenting in the emergency department of Aga Khan University Hospital, Karachi.

Overview of Literature: Cross-table cervical spine views are important in patients with signs and symptoms relating to cervical spine, but asymptomatic patients constitute a different subgroup. Accuracy of clinical examination in these patients has not been subjected to scrutiny.

Methods: All patients with blunt trauma who presented to the emergency department and underwent cross-table X-rays as part of their trauma workup were included. The X-rays were read by a radiologist not aware of the history of the patients. We recorded demographic data along with mechanism of injury, associated neck signs or symptoms whether present or not, cervical spine range of motion, associated injuries and X-ray findings. The history and examination were carried out by the on-call neurosurgery team member. The sensitivity and specificity along with negative and positive predictive value of the clinical examination were calculated. Data were analyzed using SPSS ver. 16.0.

Results: Of 50 patients with positive signs and symptoms, 4 (8\%) had positive X-rays while only 1 out of $324(0.3 \%)$ with no associated signs and symptoms had positive $\mathrm{X}$-ray findings.

Conclusions: The clinical examination is $80 \%$ sensitive and $73.98 \%$ specific in detecting true cervical spine injuries as compared to $\mathrm{C}$-spine $\mathrm{X}$-rays in alert and awake patients with blunt trauma.

Key Words: Cross table c-spine X-rays, Cervical spine trauma, Craniofacial trauma, Cervical spine fractures

\section{Introduction}

Trauma patients constitute an important subgroup that come to our emergency department. As per advanced trauma life support protocols (ATLS), all of these patients undergo a series of X-rays to detect trauma, which includes a cervical spine X-ray (cross-table), a chest X-ray, and a pelvis X-ray regardless of their clinical condition. Most of the time these X-rays do not yield any findings if the clinical exam is not significant. In addition to this, $\mathrm{X}$-rays are criticized for being expansive and exposing patients to unnecessary radiation. Although the use of cervical spine $\mathrm{X}$-rays is considered important in patients demonstrating signs and symptoms of cervical spine injury because these clinical findings are a reliable indicator of cervical spine injury [1], the same cannot be said about patients who are asymptomatic.

Received May 29, 2010; 1st Revised Jun 15, 2010; Accepted Aug 10, 2010

Corresponding author: Manzar Hussain, MBBS, FCPS

Flat No. a-30, Erum Garden Block 13/D near Hassan Square, Gulshan-e-iqbal, Karachi 75300, Pakistan

Tel: +92-343-2025927, E-mail: manzary2k@yahoo.com 
The prevalence of cervical spine injuries is increasing [2], which may be due to increased awareness and refinements in diagnostic tools. Safe and effective clearance of these cases demands vigilance on the part of the clinician [3]. Cervical spine injury is relatively rare, occurring in only $2 \%$ to $3 \%$ of patients with blunt trauma who undergo imaging studies. Evaluation of cervical spine injuries should begin in the emergency department and involve multispecialties for optimum care. Knowing which patients are at highest risk for injuries will undoubtedly influence decisions on how aggressively to pursue a potential cervical spine injury. Implementation of such guidelines will decrease time to cervical spine clearance and decrease the incidence of missed injuries [4]. There has been a controversy regarding the utility of cross-table cervical spine X-rays in awake and alert blunt trauma patients with no associated neck symptoms and no signs and no associated neurological deficits [5], despite evidence that such X-rays are usually not necessary. This is especially important in the setting of a third world country where resources are already stretched to the limit. Our aim was to assess the utility of cross-table radiography in this patient population so as to rationalize its use in trauma victims presenting at the emergency department, focusing more on the clinical examination. In recently published guidelines [6] it was recommended that patients with no distracting injury and with no neck pain or tenderness, and who have full range of motion and have no neurological deficits, should be cleared on clinical examination.

\section{Materials and Methods}

All patients who presented at the emergency department following blunt trauma, regardless of the nature of the trauma were included in the study. The study was conducted after the approval of the hospital's ethics committee. It was conducted under waiver of consent, since it involved only collection of data. Patients of all ages and both sexes were included in the study.

Patients who (1) showed neurological deficits (because these patients routinely require cervical spine X-rays) or (2) were less than 8 years of age (because the ossification of bones is not complete before that age and X-rays are hard to interpret), or (3) had severe arthritic changes (these changes make evaluation of cervical spine X-rays difficult) were excluded from the study. Those patients whose cervical spine views were inadequate were also excluded (inadequate $\mathrm{X}$-rays were defined as $\mathrm{X}$-rays that provided exposure less than cervicodorsal junction. In addition, if the exposure was poor in terms of visualization of the vertebra, the Xrays were considered inadequate. Patients with schiwora or those elderly patients who showed signs and symptoms of central cord syndrome were also excluded. All demographic data including age and sex were recorded. We recorded the mechanism of injury, the associated injuries, the clinical findings relevant to the cervical spine, including neck pain and tenderness or range of motion of cervical spine and Xrays findings. The history taking and examination were done by the on-call neurosurgery team member. All patients underwent cross table cervical spine $\mathrm{X}$-rays. The sensitivity and specificity of the clinical examination in detecting cervical spine injury was calculated using a $2 \times 2$ contingency table. The negative and positive predictive value of the clinical examination was calculated. Results were tabulated using SPSS ver. 16.0 (SPSS Inc., Chicago, IL, USA).

\section{Results}

Our patient population consisted of 374 patients. A majority of them $313(83.7 \%)$ were males. Of the 374,232 $(62.1 \%)$ less than or equal to 35 years of age while 142 (37.8\%) 35 years of age or older. Most 300 (79.72\%) were victims of road traffic accidents. Signs and symptoms that were assessed included neck pain, tenderness, and cervical range of motion. Of the 374, 100 (27.0\%) had neck symptoms, while the majority, 274 (72.97\%), had no neck symptoms. All patients regardless of their neck signs/symptoms underwent X-ray cervical spine cross-table views, as per our trauma protocol.

Amongst the 374 patients in our series, only 5 (1.33\%) had positive cross-table cervical spine X-rays. Of the 100 patients with neck signs/symptoms, 4 (4\%) had positive cervical spine X-rays for fractures. Amongst the 273 patients with no neck signs/symptoms, only $1(0.366 \%)$ had positive cross table cervical spine X-rays. Of the 374, 207 (55.4\%) had associated injuries, which included craniofacial injury $(105,28.3 \%)$, distracted by others $(85,22.9 \%)$, torso and limb injuries $(15,4.05 \%)$. Of the 374 patients, 166 (44.59\%) had no associated injuries.

The descriptions of the cervical spine injuries were as follows: one patient had C5/6 listhesis, one had a C3 fracture, two had C6/7 traumatic listhesis, and a patient who had no neck symptoms/signs had a C2 fracture involving both lateral masses. Most of the patients who had road traffic accidents or who were injured in a fall had associated neck 
Table 1. Patients with positive $\mathrm{X}$-ray findings

\begin{tabular}{lllll}
\hline \hline Age/Sex (yr) & Mechanism & X-ray findings & Neck symptoms/Signs & Associated injuries \\
\hline $27 / M$ & Rta & C5/6 listhesis & $+v e$ & -ve \\
$45 / M$ & Rta & C2 fracture involving both lateral masses & - ve & Pelvic injuries, lung contusions \\
$55 / M$ & Fall & C3 facture & + ve & -ve \\
$54 / M$ & Rta & C6/7 listhesis & $+v e$ & $-v e$ \\
$55 / M$ & Fall & C6/7 listhesis & $+v e$ & -ve \\
\hline
\end{tabular}

M: Male, Rta: Road traffic accident, +ve: Positive clinical signs/symptoms, -ve: Negative clinical signs/symptoms.

Table 2. $2 \times 2$ Contingency table for calculation of sensitivity and specificity

\begin{tabular}{lcrr}
\hline \hline & X-rays positive for fractures & X-rays negative for fractures & Total \\
\hline Neck pain/Tenderness present & 4 & 96 & 100 \\
Neck pain/Tenderness absent & 1 & 273 & 274 \\
Total & 5 & 369 & 374 \\
\hline
\end{tabular}

symptoms/signs. Only one patient with positive X-ray findings had associated injuries but no neck signs/symptoms. His associated injuries were pelvic fractures and lung contusions. Three of the 5 patients who had positive X-rays presented with a history of a road traffic accident, while two had a fall. Interestingly, the one individual who had no neck signs but had positive cervical spine X-rays had a road traffic accident. Although he had no neck signs he had associated injuries, which included lung contusions and pelvic fractures (Table 1). None of these patients needed any surgical intervention for their spinal fractures. The sensitivity and specificity of the clinical examination were $80 \%$ and $73.98 \%$ respectively (Table 2 ). The positive predictive value of the clinical examination was $20 \%$ and the negative predictive value was $98.1 \%$ (Table 2). The relevant descriptive statistics are given in Table 3 .

\section{Discussion}

Cervical spine injuries are a major cause of morbidity and mortality following blunt trauma. Cervical spine clearance constitutes an important part of the management of trauma patients. But the contradictory literature makes it very difficult for a health care professional to choose the most appropriate clearance plan [7]. Before the advent of computed tomography (CT) scans, X-rays were considered the investigational method of choice for all those patients who needed clearance of the cervical spine [8]. But, as CT scan quality and availability improved, it started replacing cross table views [8] .

The fear of missing a cervical spine injury, which could
Table 3. Relevant disccrptive statistics $(n=374)$

\begin{tabular}{lc}
\hline \hline Characteristics & No. \\
\hline Male & 313 \\
Female & 61 \\
Age (mean \pm SD) & \\
Mechanism & 32.360 \\
$\quad$ Rta & 54 \\
Fall & 20 \\
Others & \\
Clinical findings & 100 \\
Clinical findings present & 274 \\
Clinical findings absent & \\
X-ray findings & 5 \\
X-ray findings present & 369 \\
X-ray findings absent & \\
\hline
\end{tabular}

SD: Standard deviation, Rta: Road traffic accident.

have devastating consequences, has led to the indiscriminate use of cervical spine X-rays. As part of ATLS, all patients undergo a trauma series of X-rays, which include a cervical spine X-ray (cross-table), a chest X-ray chest, and a pelvis X-ray regardless of their clinical condition. Most of the time these $\mathrm{X}$-rays do not yield any findings in patients with no clinical evidence of cervical spine injury. These $\mathrm{X}$ rays are criticized for being expansive and exposing patients to unnecessary radiation. Moreover, it not only adds to the patients' discomfort, but also, requires the mobilization of resources that at times can put too much burden on the already stretched resources of radiology departments. Some have argued [9] that the clinical examination on its own cannot be used reliably to clear the cervical spine in patients who have no cervical spine signs/symptoms, who are not 
intoxicated, and who have no distracting injuries.

Although nowadays CT scans are frequently utilized as a diagnostic tool for cervical spine clearance, in a third world country like ours (Pakistan), where a large number of patients belong to low socioeconomic groups, and the health budget does not allow much expenditure for diagnostics, cervical spine X-rays constitute an important part of the neurosurgeon's armamentarium [10]. Even in our setups, CT scans may still be an important tool in those patients who have a very high suspicion level of cervical injury or are obtunded or non-cooperative [11]. At present there is insufficient data to suggest the routine use of CT scans in patients who have a low likelihood of having cervical spine trauma and cervical spine $\mathrm{X}$-rays may still be important in these patients [11].

Cervical spine injuries are a part of craniofacial trauma and usually do not occur in isolation. In an article by Beirne et al. [12], this association was explored and it was found that patients with craniofacial trauma may have associated cervical spine injuries. Similar observations were made by Merritt and Williams [13]. It is well known that 3 views for $\mathrm{C}$-spine clearance are more useful than a single view for clearance. In a comparative study, comparing a single cervical spine view with a 3-view radiographic screening, it was concluded by West et al. [14] that 3 views were better than a single view, in terms of detecting cervical spine injuries.

There has been a controversy regarding the utility of cross-table cervical spine $\mathrm{X}$-rays in awake and alert blunt trauma patients with no associated neck symptoms and signs and no associated neurological deficits. Recently the eastern association of spinal trauma [5] has put forward a protocol which states that patients who have no neck signs or symptoms, are not intoxicated, and have no neurological deficits, can be safely cleared for cervical injury without subjecting them to cervical spine X-rays. This protocol is criticized because if a cervical spine injury is missed, then the cost may be devastating both for the patient and the clinician. This fear has even prompted the use of CT scans of the cervical spine for cervical spine clearance. However, in a third world country like ours, cost effectiveness is a major concern both for the clinicians and the patient`s family.

In a series done prospectively by Valmahos et al. [15] who looked at 549 patients, the authors concluded that where there was no clinical evidence of cervical spine injury, none had positive X-rays. In another study, Ersoy et al. [16] retrospectively reviewed 267 patients. It was con- cluded patients who had no local signs in the cervical spine, or neurological deficits, or distraction injuries, and who were not intoxicated and were awake and alert, could be safely screened clinically. The same conclusion was drawn in recently published guidelines by the eastern association for the surgery of trauma. In the year 2000, The National Emergency X-Radiography Utilization Study Group [17], published a study that was done prospectively. It was an observational study involving 21 centers in the United States. More than 34,000 patients were included. Of the 34,000 , only 818 patients had cervical spine injury, and $99 \%$ (all but 8 of the 818 ) were identified using a clinical examination based on following factors: non-tender posterior midline cervical spine, no focal neurological deficit, normal level of alertness, no evidence of intoxication and no obvious distraction injury. Only 2 of the 8 patients were considered significant. Based on these results, the conclusion was drawn that patients without the above mentioned clinical findings can be safely excluded from the cervical spine X-ray protocol. The effectiveness of this protocol has not only been tested in adults but it is also equally effective in children [18].

Gonzalez et al. [19] performed a prospective evaluation of 2,176 consecutive trauma patients, of whom $33(1.6 \%)$ had a cervical spine injury. Of the 33 cervical spine injuries, only 3 had negative clinical examinations. These 3 patients were found to have a $\mathrm{C} 2$ spinous process fracture and $\mathrm{C} 6-\mathrm{C} 7$ body fractures, and a $\mathrm{C} 1$ lamina fracture along with $\mathrm{C} 6-\mathrm{C} 7$ body fractures. It was concluded that the clinical examination of the neck could reliably rule out significant cervical spine injury in the awake and alert blunt trauma patient.

Our study also supports the notion that most of our patients who had cervical spine injuries also had neck findings, and patients who had no clinical findings also have negative X-rays. However, it must be remembered that cervical spine X-rays may still miss injuries [20] due to inadequate exposure [21] and this must be balanced against the utility and cost of CT scans.

\section{Conclusions}

The clinical examination is $80 \%$ sensitive and $73.98 \%$ specific in detecting true cervical spine injuries as compared to $\mathrm{C}$-spine X-rays in alert and awake patients with blunt trauma. 


\section{REFERENCES}

1. Mahadevan S, Mower WR, Hoffman JR, Peeples N, Goldberg W, Sonner R. Interrater reliability of cervical spine injury criteria in patients with blunt trauma. Ann Emerg Med 1998;31:197-201.

2. Wang MC, Pintar F, Yoganandan N, Maiman DJ. The continued burden of spine fractures after motor vehicle crashes. J Neurosurg Spine 2009;10:86-92.

3. O'Dowd JK. Basic principles of management for cervical spine trauma. Eur Spine J 2010;19 Suppl 1:S18-22.

4. Agrawal A. Cervical spine clearance: a review and understanding of the concepts. JNMA J Nepal Med Assoc 2008;47:244-50

5. Eastern Association for the Surgery of Trauma (EAST) guidelines. Determination of cervical spine stability in trauma [Internet]. Chicago: Eastern Association for the Surgery of Trauma, 2009 [Cited 2010 May 28]. Available from: http://www.east.org.

6. Como JJ, Diaz JJ, Dunham CM, et al. Practice management guidelines for identification of cervical spine injuries following trauma: update from the eastern association for the surgery of trauma practice management guidelines committee. J Trauma 2009;67:651-9.

7. Tins B, Cassar-Pullicino V. Controversies in "clearing" trauma to the cervical spine. Semin Ultrasound CT MR 2007;28:94-100.

8. Fisher A, Young WF. Is the lateral cervical spine x-ray obsolete during the initial evaluation of patients with acute trauma? Surg Neurol 2008;70:53-7.

9. Duane TM, Dechert T, Wolfe LG, Aboutanos MB, Malhotra AK, Ivatury RR. Clinical examination and its reliability in identifying cervical spine fractures. J Trauma 2007;62: 1405-8.

10. Shaukat A, Ahmad G, Anjum MN. Cervical spine trauma $\mathrm{X}$-ray interpretation pictorial review. Pak J Radiol 2003;15:26-37.

11. Holmes JF, Akkinepalli R. Computed tomography versus plain radiography to screen for cervical spine injury: a meta-analysis. J Trauma 2005;58:902-5.

12. Beirne JC, Butler PE, Brady FA. Cervical spine injuries in patients with facial fractures: a 1-year prospective study. Int J Oral Maxillofac Surg 1995;24(1 Pt 1):26-9.

13. Merritt RM, Williams MF. Cervical spine injury complicating facial trauma: incidence and management. Am J Otolaryngol 1997;18:235-8.

14. West OC, Anbari MM, Pilgram TK, Wilson AJ. Acute cervical spine trauma: diagnostic performance of single-view versus three-view radiographic screening. Radiology 1997;204:819-23.

15. Velmahos GC, Theodorou D, Tatevossian R, et al. Radiographic cervical spine evaluation in the alert asymptomatic blunt trauma victim: much ado about nothing. J Trauma 1996;40:768-74.

16. Ersoy G, Karcioğlu O, Enginbas Y, Eray O, Ayrik C. Are cervical spine $\mathrm{X}$-rays mandatory in all blunt trauma patients? Eur J Emerg Med 1995;2:191-5.

17. Hoffman JR, Mower WR, Wolfson AB, Todd KH, Zucker MI. Validity of a set of clinical criteria to rule out injury to the cervical spine in patients with blunt trauma. National Emergency X-Radiography Utilization Study Group. N Engl J Med 2000;343:94-9.

18. Anderson RC, Scaife ER, Fenton SJ, Kan P, Hansen KW, Brockmeyer DL. Cervical spine clearance after trauma in children. J Neurosurg 2006;105(5 Suppl):361-4.

19. Gonzalez RP, Fried PO, Bukhalo M, Holevar MR, Falimirski ME. Role of clinical examination in screening for blunt cervical spine injury. J Am Coll Surg 1999;189:1527.

20. Besman A, Kaban J, Jacobs L, Jacobs LM. False-negative plain cervical spine $x$-rays in blunt trauma. Am Surg 2003;69:1010-4.

21. Gale SC, Gracias VH, Reilly PM, Schwab CW. The inefficiency of plain radiography to evaluate the cervical spine after blunt trauma. J Trauma 2005;59:1121-5. 